\title{
The design of the superconducting wiggler power supply system for HEPS-TF
}

\author{
Xu Wang ${ }^{1,2}$ (D) $\cdot$ Fengli Long $^{1} \cdot$ Yao Gao $^{1}$
}

Received: 9 December 2018 / Revised: 18 March 2019 / Accepted: 18 March 2019 / Published online: 9 April 2019

(c) The Author(s) 2019

\begin{abstract}
Purpose This paper describes the design of power supply system for superconducting wiggler in the project of high energy photon source-test facility. It includes three sets of high precision DC stabilized current power supplies for superconducting magnet excitation and three sets of quench protection assembly (QPA). A special magnetic load which has a very large time constant is studied in this paper. In this paper, a stable superconducting excitation power supply is developed, which can adjust the response quickly under the condition of very large load time constant. Meanwhile, the energy dissipation circuit of the QPA is integrated into the power supply in an active quenching protection mode to accelerate the energy release of the superconducting magnet and protect the superconducting magnet.

Method The main circuit topology, control algorithm, quenching protection circuit and interlocking logic function are designed for the superconducting magnet with large inductance. The upper computer controls the switch of the three main power circuits through the serial port, sets the output current and adjusts the parameters according to the load.

Conclusion Through a series of scientific and rigorous experiments, the rationality of superconducting wiggler magnet power supply scheme is demonstrated. The output current stability test and output voltage ripple test of the power supply meet or exceed the requirements of the magnet system.
\end{abstract}

Keywords HEPS-TF · Superconducting magnet power supply $\cdot$ Quench protection assembly

PACS 29.20.-c $\cdot 84.71 . \mathrm{Ba} \cdot 07.05 . \mathrm{Hd}$

\section{Introduction}

The high energy photon source (HEPS) is the fourth generation of synchrotron radiation source to be established in China. The beam energy is $6 \mathrm{GeV}$, and the emittance is less than $0.1 \mathrm{~nm} \cdot \mathrm{rad}$. High energy photon source-test facility (HEPS-TF) is the research and development stage of HEPS. Superconducting $3 \mathrm{~W} 1$ magnet is the first self-developed superconducting wiggler magnet in China and one of the research contents of the HEPS-TF insertion device system. The superconducting magnet power supply and quenching protection assembly (QPA) are developed for the

Xu Wang

wangxu@ihep.ac.cn

1 Institute of High Energy Physics, Chinese Academy of Sciences, Beijing 100049, China

2 University of Chinese Academy of Sciences, Beijing 100049, China nonlinearity of superconducting magnet inductance to output current [1]. The energy dissipation circuit of the quench protection circuit is integrated into the superconducting excitation power supply in an active protection mode. After the quench of superconductivity, the power supply is quickly turned off and the magnet is connected to the dissipation circuit through IGBT switch. And in the process of current attenuation, the voltage at both ends of the coil can be maintained at $1000 \mathrm{~V}$. The power supply is divided into two parts: the power supply part and the quench protection circuit part. The power supply mainly provides a stable excitation current for the load. In order to avoid damaging the superconducting magnet, we need to use the quench protection circuit to reduce the current in the magnet to the safe value within a short time and transfer its energy to the extraction resistor for consumption. Make the superconducting magnet quickly from the superconducting state into the resistance state so as to protect the expensive superconducting magnet [2]. 


\section{Superconducting magnet power supply}

The superconducting $3 \mathrm{~W} 1$ wiggler magnet power supply includes three sets of high precision DC current stabilizing power supply and three sets of quenching protection system. After receiving the quenching signal, start the protection circuit to disconnect the magnet power supply and set the current value to the initial state. The main technical specifications of superconducting magnet power supply are shown in Table 1.

The main circuit of the power supply is divided into two stages. The first stage is the stabilized DC source, and the low-frequency voltage ripple is suppressed to $0.1 \%$. The latter stage is the chopper for steady current output [3,4]. The main circuit of 580 A power supply adopts the phaseshifting soft switch full-bridge conversion circuit, which is shown in Fig. 1. The 65 A power supply adopts hard switch full-bridge structure. The hard switch full-bridge conversion circuit is shown in Fig. 2 [5].

Table 1 Main technical specifications of superconducting magnet power supply

\begin{tabular}{lll}
\hline Power supply & The main coil & $\begin{array}{l}\text { The correction } \\
\text { coil }\end{array}$ \\
\hline Quantity & 1 & 2 \\
Rated output current/(A) & 580 & \pm 65 \\
Magnet design current/(A) & 400 & \pm 20 \\
Rated output voltage/(V) & 7 & \pm 5 \\
Rated output power/(KW) & 4.06 & 0.325 \\
Current stability/(ppm) & $\leq 50$ & $\leq 50$ \\
\hline
\end{tabular}

\section{Load time constant}

For any accelerator magnet power supply, its load characteristics are the basis of power closed-loop control. For the normal conducting magnet, we generally adopt proportional integral differential (PID) control as the closed-loop control algorithm, and the core design of the current loop integral time constant mainly depends on the load time constant $[6,7]$. For the superconducting magnet, its load time constant is much larger the normal one.

In the design of digital PID controller, it is impossible to set the time constant of the current loop as the same as the load time constant, so it is easy to cause overshoot of the system. In high-power superconducting magnet power supply, due to the strict requirements of beam loss, it is required to accurately control the current of power supply and quickly respond to disturbance in the ramping process. Therefore, the special PID closed-loop tracking and adjusting characteristics of the independent controllable algorithm is designed to overcome the controlled object which is difficult to both track and adjust the characteristics of the shortcomings. The control block diagram is shown in Fig. 3 [8, 9].

The tracking transfer function is shown in Eq. (1):

$\frac{W(k)}{Y(k)}=\frac{z^{-\mathrm{d}} \cdot \mathrm{B} \cdot \mathrm{T}}{\mathrm{A} \cdot \mathrm{S}+\mathrm{R} \cdot \mathrm{B} \cdot z^{-\mathrm{d}}}$

And the regulation transfer function is shown in Eq. (2):

$\frac{Y(k)}{E}=\frac{\mathrm{A} \cdot \mathrm{S}}{\mathrm{A} \cdot \mathrm{S}+\mathrm{R} \cdot \mathrm{B} \cdot z^{-\mathrm{d}}}$

This special PID algorithm makes it possible to obtain the desired tracking behaviour (following the reference)

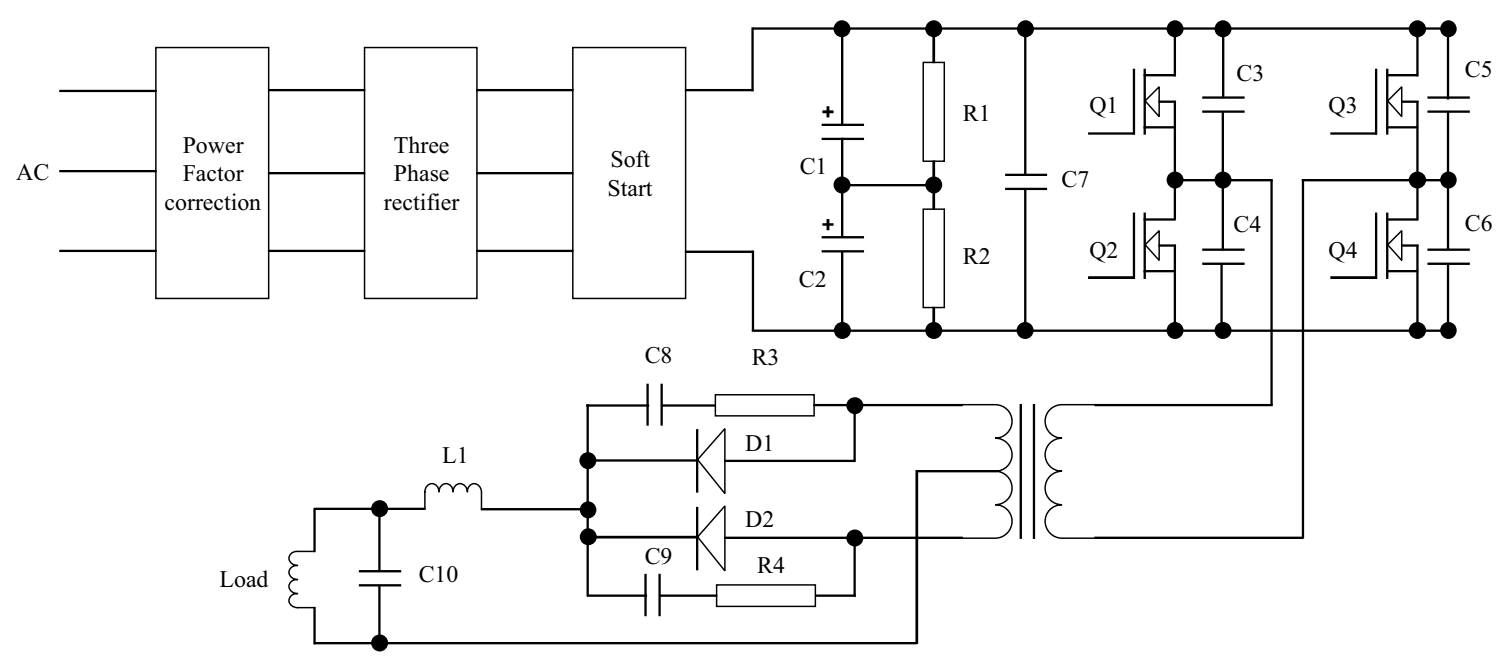

Fig. 1 Soft switch full-bridge conversion circuit 


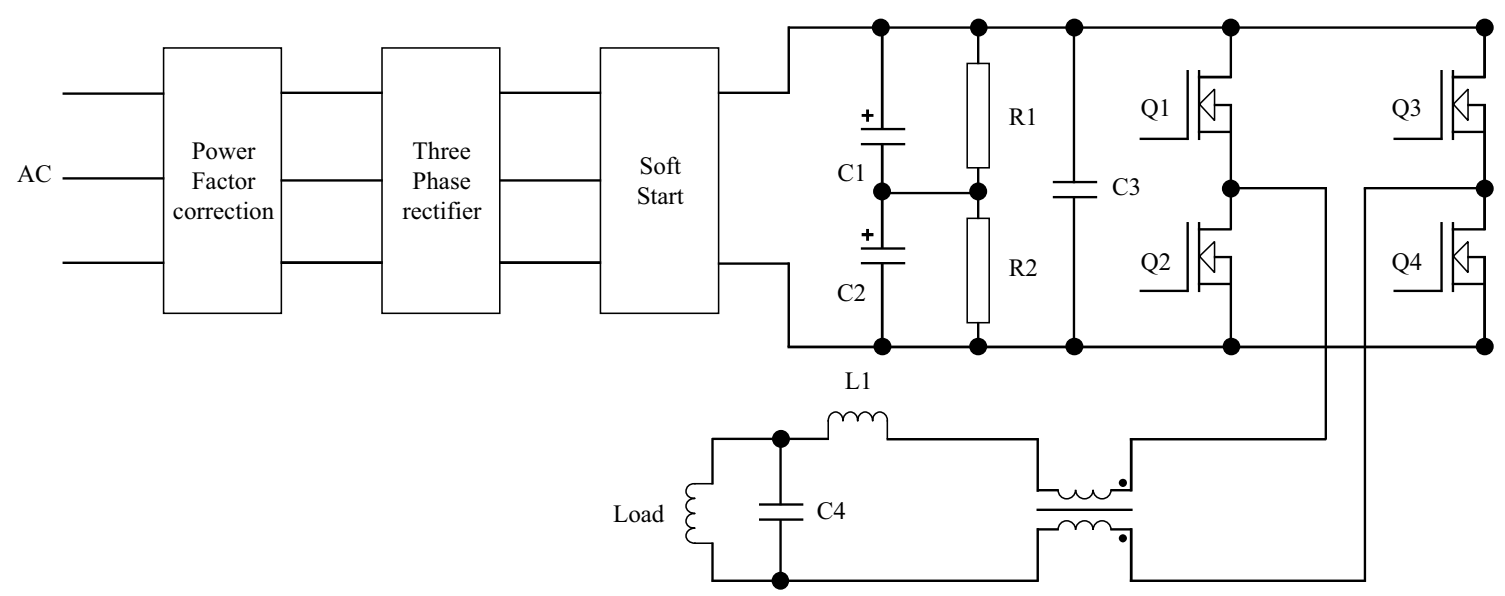

Fig. 2 Hard switch full-bridge conversion circuit

Fig. 3 Control algorithm block diagram

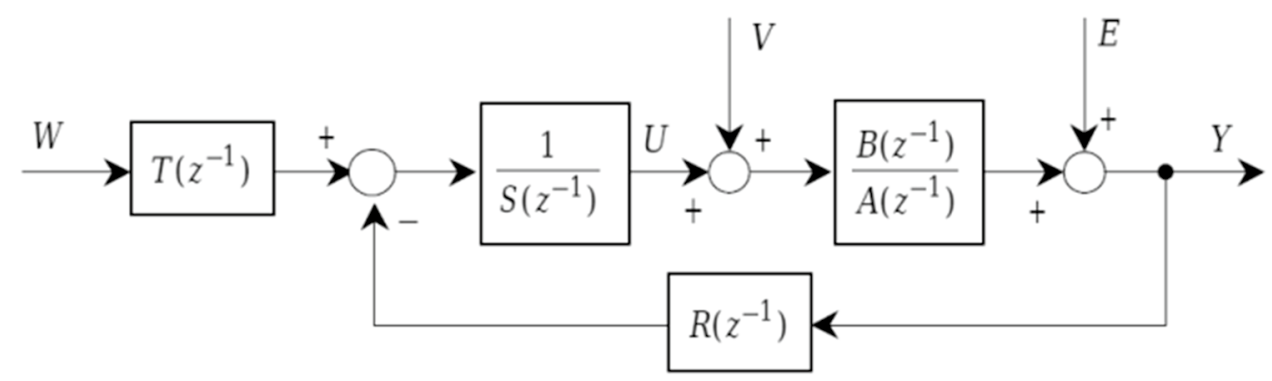

independent of the desired regulation behaviour (rejection of a disturbance) [10].

\section{Quench protection assembly (QPA)}

Superconducting magnets are very sensitive to current fluctuations, and excessive current fluctuations can cause the superconducting coil to lose its superconductivity. This seriously affects the normal operation of superconducting magnets and even destroys them. Therefore, the stability of the output current, ripple, adjustment accuracy and dynamic response ability of the superconducting excitation power supply are strictly required. These applications require that the output current of the power supply has a high stability and can quickly realize quench protection. The maximum working current of the main coil of the superconducting $3 \mathrm{~W} 1$ wiggler magnet is $400 \mathrm{~A}$, the energy of the magnet is $288 \mathrm{~kJ}$, and the inductance is $3.6 \mathrm{H}$. The energy dissipation circuit of quenching protection circuit is integrated into the superconducting excitation power supply. After quenching, the power supply is quickly turned off, and the energy dissipation circuit is connected through IGBT switch [11]. The withstand voltage of IGBT is greater than $1500 \mathrm{~V}$, and the voltage at both ends of the coil can be maintained at $1000 \mathrm{~V}$ during the current attenuation process. The main technical
Table 2 Main technical specifications of the quenching protection circuit

\begin{tabular}{llll}
\hline Power supply & $\begin{array}{l}\text { Magnet coil } \\
\text { inductance/(H) }\end{array}$ & Resistance/( $\Omega)$ & $\begin{array}{l}\text { The highest } \\
\text { voltage/(V) }\end{array}$ \\
\hline The main coil & 3.6 & 2.5 & 1000 \\
The correction coil & 2.38 & 1.0 & 450 \\
\hline
\end{tabular}

specifications of the quenching protection circuit are shown in Table 2.

When the quenching protection circuit receives the fault signal from the detection circuit, the quenching protection circuit must reduce the current in the superconducting coil to a safe current value in a very short time. This process is accomplished by switching on an energy extraction resistors and connecting a crowbar circuit and cutting off the power supply at the same time through the quenching protection system. The schematic diagram of unipolar quenching protection circuit (QPA) is shown in Fig. 4.

When the power supply receives the quenching signal, IGBT is turned off in the energy release cabinet. The energy in the coil is released through two different energy release loops, namely the quenching box and the energy release box, so as to rapidly release energy and protect the coil. At this point, SCR is closed. If the IGBT of QPA fails to be 


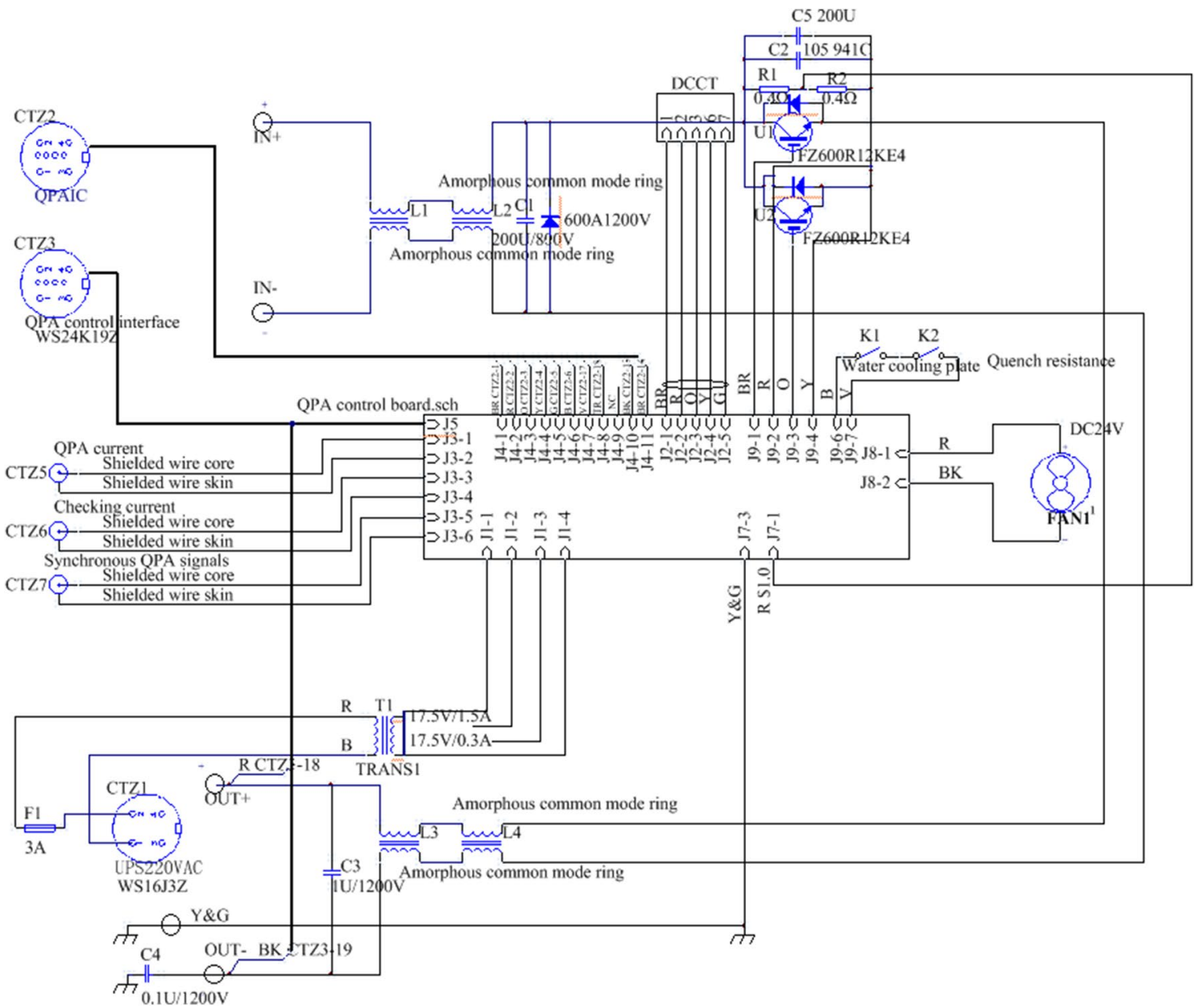

Fig. 4 Schematic diagram of unipolar quench protection circuit

switched on in time, the back-up SCR circuit can also realize energy release. After IGBT turn off, the energy storage capacitor bank is charged to $1100 \mathrm{~V}$, and then the superconducting coil energy is released through rapid conduction. The open circuit voltage of $1100 \mathrm{~V}$ can not only prevent the misoperation of SCR, but also ensure the timely release of energy, thus protecting the coil. The schematic diagram of the back-up SCR circuit is shown in Fig. 5.

\section{Prototype and system test}

The main coil magnet power supply part and QPA are separated in the different crates, and the correction coil power supply part and the quenching protection circuit part are in the same crate. The inner structure of the main coil magnet power supply and the quenching protection circuit is shown in Fig. 6, and the inner structure of the superconducting correction coil magnet power supply and quenching protection circuit is shown in Fig. 7.

The upper computer can control the main circuit switch of the three power supplies through the serial port, set the output current and adjust the parameters according to the load. In order to facilitate the field operation, the touch screen function of man-machine interaction is also designed [12]. The touch screen can realize the switch of the main circuit of the power supply and the function of setting the output current and can clearly view the power supply fault information in the fault display interface [13], which is convenient for the maintenance staff. The acceptance test results are shown in Fig. 8. The energy release time is about $2000 \mathrm{~ms}$.

Channel 1 is the voltage change during QPA response (the yellow line). Channel 2 is the voltage change at the output (the blue line). And the output terminal voltage from 0 to $-1000 \mathrm{~V}$ takes about $10 \mathrm{~ms}$, as shown in Fig. 9.

After receiving the quenching signal, the QPA response time of the power supply is $52 \mu$ s, as shown in Fig. 10. 


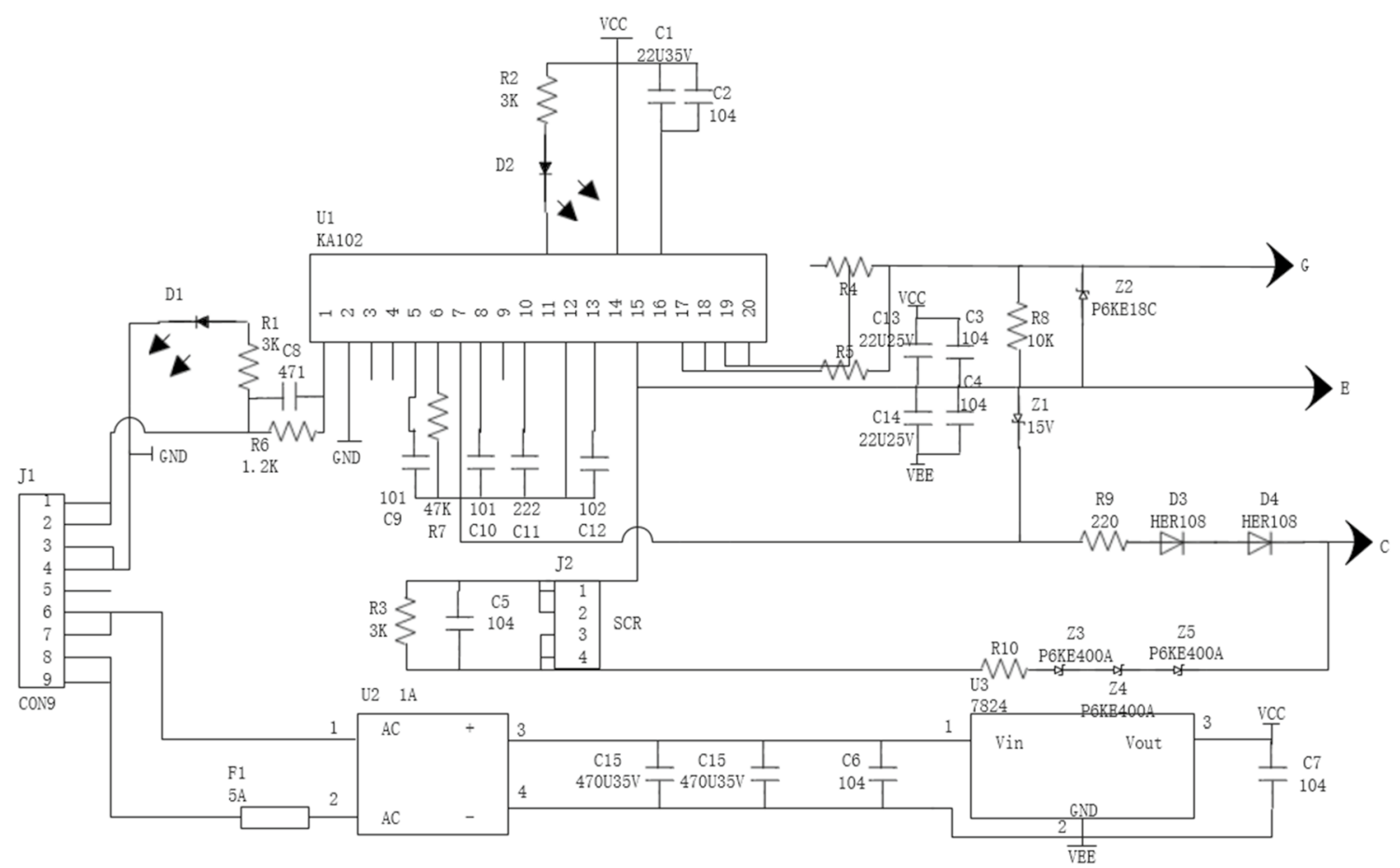

Fig. 5 Schematic diagram of the back-up SCR circuit

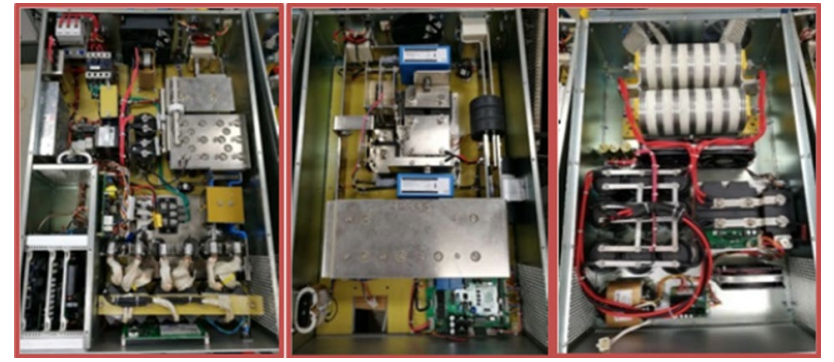

Fig. 6 The inner structure of superconducting main coil magnet power supply and quenching protection circuit

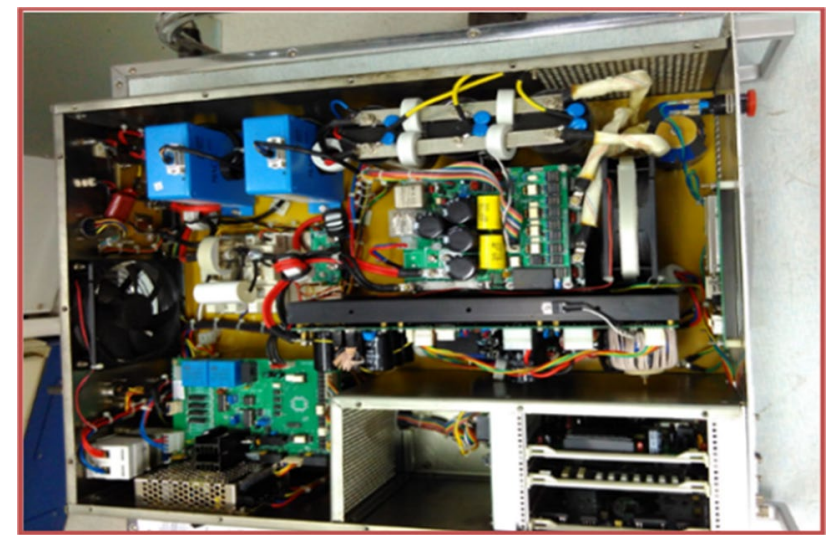

Fig. 7 The inner structure of superconducting correction coil magnet power supply and quenching protection circuit

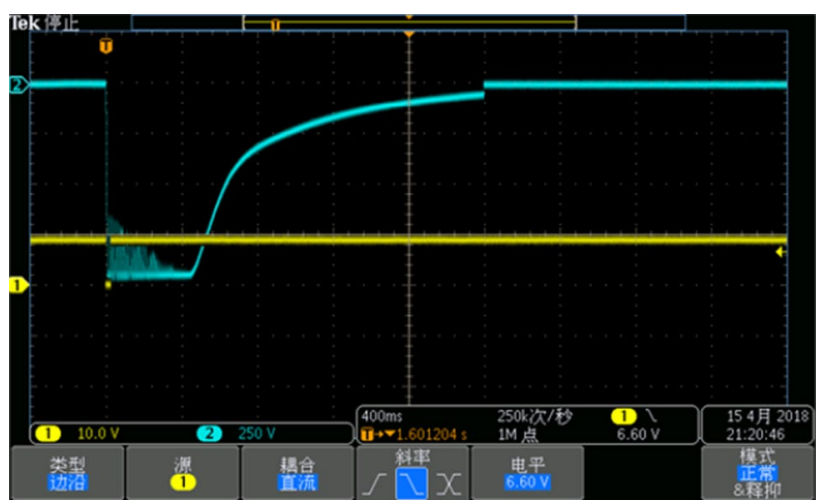

Fig. 8 Energy release time

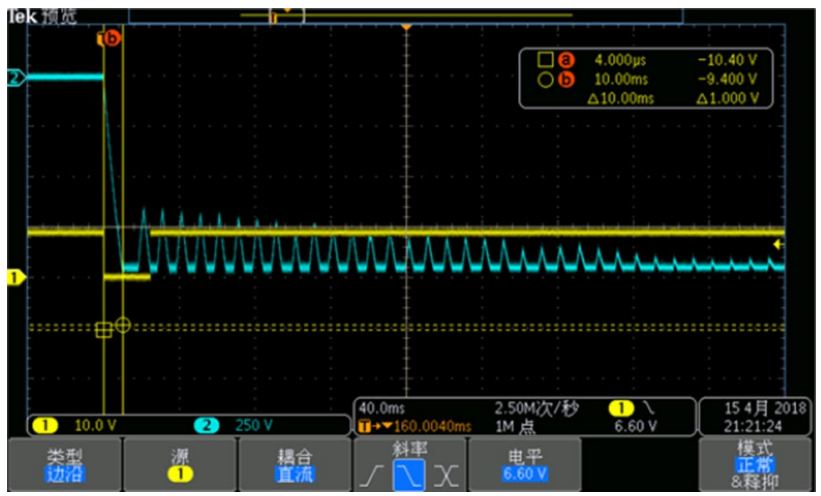

Fig. 9 The output voltage is from $0 \mathrm{~V}$ to $-1000 \mathrm{~V}$ 


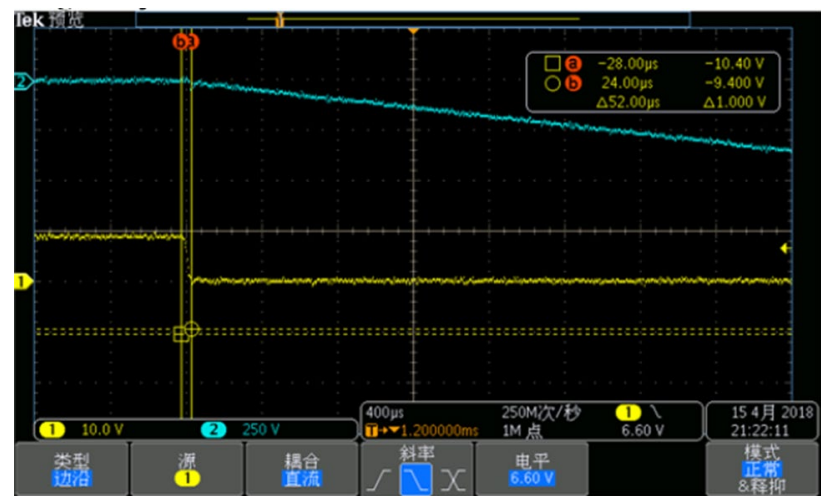

Fig. 10 The response time of quench protection assembly

The power supply system meets the design requirements of magnet system.

\section{Conclusion}

Superconducting magnet power supply is a very important power supply, which is one of the key equipments of accelerator technology in the field of basic science research. This paper introduces the design of power supply system for superconducting wiggler in the high energy photon sourcetest facility (HEPS-TF) and studies the main circuit topology, control algorithm, quench protection circuit and interlocking logic function of the superconducting magnet power supply with very large load time constant. All three kinds of power supply can achieve the purpose of controlling the main circuit switch of power supply, setting output current and adjusting parameters according to the load. Through a series of scientific and rigorous experiments, the rationality of superconducting wiggler magnet $3 \mathrm{~W} 1$ power supply technology scheme is demonstrated. The output current stability test and output voltage test of the power supply meet or even exceed the requirements of the magnet system.

Acknowledgements The authors thank the Accelerator Centre of Institute of High Energy Physics for financial support. Also thanks to the experimental conditions and experimental guidance provided by the power supply group of IHEP.

Open Access This article is distributed under the terms of the Creative Commons Attribution 4.0 International License (http://creativeco mmons.org/licenses/by/4.0/), which permits unrestricted use, distribution, and reproduction in any medium, provided you give appropriate credit to the original author(s) and the source, provide a link to the Creative Commons license, and indicate if changes were made.

\section{References}

1. F.L. Long, Research of intelligent control for high precision stabilized current power supply, Beijing: doctoral dissertation of graduate school of Chinese academy of sciences, 2006

2. F.L. Long, The technical agreement of superconducting $3 \mathrm{~W} 1 \mathrm{wig}-$ gler magnet power supply system. HEPS, Institute of High Energy Physics, Chinese Academy of Sciences, 2017

3. R. Lambiase, B. Oerter, J. Smith, Power supply control for the spallation neutron source, EPAC'00, Vienna, 06/2000

4. S. Peng, R. Lambiase, B. Oerter, J. Smith, SNS standard power supply interface, ICALEPCS'O1, California, 11/2001

5. M. Wang et al., in Real time PWM control converters using three elements polynomial regulators, IEEE, 1992

6. I. Landau, The RST digital controller design and applications. Control Eng. Pract. 6, 155-165 (1998)

7. I. Barnett et al., Developments in the high precision control of magnet currents for LHC, PAC'99, New York, NY, USA, p. 3743

8. P. Proud lock, in Proceedings of Electrical powering strategy of LHC, EPAC'96, June 1996, paper MOP021

9. I. Barnett et al., in Proceedings of A Strategy for controlling the LHC magnet currents, EPAC'96, June 1996, paper MOP020G, p. 2317

10. G.Q. King et al., in Proceedings of the all-digital approach to LHC power converter current control, ICALEPCS'01, San Jose, CA, USA, paper THBT004, pp. 453-455

11. Y.Y. Wang, Fast and high precision IGBT switching pulse/Dc stabilized current supply. J. Electr. Technol. 20(10), 15-18 (2003)

12. J. Liu, C. H. Wang, J. Wang, BEPCII superconducting magnet power supply magnetic measurement control system, in Proceedings of the 12th Beijing electron-positron collider annual conference

13. J. Naik, Serial interface for debugging memory based subsystems, Apogee Labs, 01/11/2001 